\title{
Nivolumab in the Treatment of Recurrent/Metastatic Squamous Cell Carcinoma of the Head and Neck (RM-SCCHN): A Report of 16 Cases at a Single Institution
}

\author{
Tomoko Yamazaki $^{1}$ jiro Aoi ${ }^{2}$ Kazutaka Kishimoto ${ }^{2}$ Satoshi Saijo ${ }^{2}$ Keitaro Fujii ${ }^{2}$ Takayuki Imai ${ }^{2}$ \\ Yukinori Asada ${ }^{2}$ Kazuto Matsuura² \\ ${ }^{1}$ Division of Head and Neck Medical Oncology, Miyagi Cancer Center, \\ Medeshima, Natori, Miyagi, Japan \\ 2 Division of Head and Neck Medical Surgery, Miyagi Cancer Center, \\ Medeshima, Natori, Miyagi, Japan \\ Address for correspondence Tomoko Yamazaki, MD, DDS, PhD, \\ Division of Head and Neck Medical Oncology, Miyagi Cancer Center, \\ 47-1 Nodayama, Shiote, Medeshima, Natori, Miyagi, 981-1293, Japan \\ (e-mail: tomoko-yamazaki@miyagi-pho.jp).
}

Int J Pract Otolaryngol 2019;2:e7-e10.

\begin{abstract}
Background Nivolumab, approved in Japan for platinum-refractory recurrent/metastatic squamous cell carcinoma of the head and neck (RM-SCCHN) in 2017, is of uncertain cost-effectiveness.

Patients and Methods We reviewed the data of 16 patients with platinum-refractory RM-SCCHN treated with nivolumab monotherapy, $3 \mathrm{mg} / \mathrm{kg}$ every 2 weeks, between April 2017 and February 2018.

Results All 16 patients were male. The number of previous treatments was 1, 2, and 3 in 1,5 , and 10 patients, respectively. All patients had been previously treated with regimens that included platinum, and 15 patients had previously received cetuximab. The best response rate was a partial response in two patients. Stable disease occurred in 11 patients and disease progression occurred in 2 patients. The disease control rate was $81.2 \%$. Median follow-up time was approximately 8.7 months, and median progression-free survival (PFS) was 2.1 months. Adverse events (AEs) $\leq$ Grade 3 included pneumonitis and rash in $38 \%$, pruritus in $31 \%$, fatigue in $25 \%$, and kidney dysfunction and endocrine disorder in 12\% each. AEs > Grade 3 included pruritus in $12 \%$, and pneumonitis in $6 \%$. Drug discontinuation was requested by patients' clinicians for disease progression in seven patients and Grade $3 \mathrm{AEs}$ in three. Following nivolumab

Keywords treatment, seven patients received salvage treatment.

- head and neck cancer

- platinum

- drug

Conclusion Nivolumab showed some efficacy in disease control, but PFS was low. The AE rate was acceptable, with no Grade 4 or 5 . If patient selection can be fine-tuned, treatment with this agent may become cost-effective.
\end{abstract}

\section{Introduction}

Head and neck cancer accounts for $>600,000$ new cases per year, with $>300,000$ deaths annually worldwide, indicating a poor prognosis. ${ }^{1}$ Patients with recurrent or metastatic squamous cell carcinoma of the head and neck (RM-SCCHN) have an especially poor prognosis, with a median survival of

received

November 12, 2018

accepted

January 18, 2019
DOI https://doi.org/

10.1055/s-0039-1685175. ISSN 2569-1783. approximately 6 months if untreated. Many of the adverse events (AEs) of therapy for this disease involve the immune system, and care by a multidisciplinary team is essential. Platinum-based chemotherapy with cetuximab is first-line chemotherapy in RM-SCCHN. ${ }^{2,3}$ A phase III trial showed that nivolumab treatment resulted in improved overall survival (OS), progression-free survival (PFS), and response rate (RR)

Copyright $\odot 2019$ Georg Thieme Verlag License terms KG Stuttgart · New York

(요 $\Theta \circledast$ 
with improved quality of life (QOL) compared with standard chemotherapy for platinum-refractory disease (cetuximab, methotrexate, and docetaxel). ${ }^{4-6}$

In Japan, nivolumab was approved for treatment of platinum-refractory SCCHN in March 2017.

We conducted a retrospective study of the 16 patients treated in our institution.

\section{Patient and Methods}

We examined the cases of 16 patients (all male; mean age: 69 years, range: 48-75 years) with RM-SCCHN, who were treated at our institution from April 2017 to January 2018. Four, six, and six patients had an Eastern Cooperative Oncology Group (ECOG) performance status of 0,1 , and 2, respectively. The primary lesions were oropharyngeal in four patients, hypopharyngeal in eight patients, oral cavity in two, larynx in one, and maxilla in one. All tumors were squamous cell carcinoma. All had a smoking history; two were current smokers and 14 were former smokers. The Brinkman index of all 14 patients was $>100$.

Two patients had unresectable local lymph node involvement, two patients had local recurrences, eight patients had distant metastases, and four patients had locoregional spread plus distant metastases.

A total of 14 patients (87.5\%) had undergone radiotherapy. Systemic chemotherapy included one regimen in one patient, two regimens in five patients, and three regimens in 10 patients. All patients had received platinum chemotherapy between one and three times and 15 patients had also received cetuximab. All experienced recurrence within 6 months.

Pretreatment evaluation consisted of complete history and physical examination, complete blood counts, liver and renal function tests, chest X-rays, and electrocardiograms (ECGs). All patients underwent a computed tomography (CT) scan and fiberoptic endoscopy of the head and neck. Tumor staging was performed based on CT findings using the TNM classification of the UICC 7th edition. Nivolumab monotherapy was administered at a dose of $3 \mathrm{mg} / \mathrm{kg}$ every 2 weeks.

Chemotherapy-related toxicities were quantified using the National Cancer Institute Common Toxicity Criteria (Version 4.0).

Follow-up time for each patient was calculated as the time from the start of treatment to March 31, 2018. Tumor responses was assessed according to response evaluation criteria in solid tumors (RECIST), version 1.1, every 6 weeks.

Survival curves were generated using the Kaplan-Meier method. Safety and efficacy analysis was conducted on an intention-to-treat basis, defined as all patients who received at least one dose of nivolumab. PFS was calculated from the date of first administration of nivolumab to disease progression, subsequent changes in therapy (for example, chemotherapy), or death, whichever came first.

OS was determined from the date of the first administration of chemotherapy to the date of death or the last confirmed date of survival. Statistical data were generated using a commercial software (SPSS statistics 21[R], SPSS, Inc., Chicago, Illinois, United States).

\section{Results}

Patient characteristics are summarized in - Table $\mathbf{1}$.

The median number of infusions administered was 5 (range: 2-11). Of the 16 patients, 13 patients needed to discontinue nivolumab because of progressive disease in 10 patients and Grade 3 AEs in three patients, including Grade 3 pneumonitis in one patient and Grade 3 rash in two patients. These AEs were unresponsive to intravenous steroids, and their clinicians requested the discontinuation of nivolumab therapy.

After discontinuation of nivolumab, 5 of 13 patients received further chemotherapy, one with paclitaxel and cetuximab and four patients with S-1.

\section{Efficacy}

The median follow-up time was 8.3 months (range: 0.6-9.4 months). The median PFS was 2.1 months (95\% confidence interval [CI]: 1.6-4.9). The median OS was 8.7 months (95\% CI: 8.1-9.4). The PFS at 6 months was $14 \%$. OS in patients with PD-L1 $\geq 1 \%$ was 3.8 months versus 6.7 months in those with PD-L1 $<1 \%$.

Table 1 Characteristics of the 16 patients

\begin{tabular}{|c|c|}
\hline Characteristics & $n$ \\
\hline \multicolumn{2}{|l|}{ Age $(y)$} \\
\hline Median & 69 \\
\hline Range & $48-75$ \\
\hline \multicolumn{2}{|l|}{ Sex } \\
\hline Male & 16 \\
\hline Female & 0 \\
\hline \multicolumn{2}{|l|}{ ECOG performance score } \\
\hline 0 & 4 \\
\hline 1 & 6 \\
\hline 2 & 6 \\
\hline \multicolumn{2}{|l|}{ Site of primary tumor } \\
\hline Oropharynx & 4 \\
\hline Hypopharynx & 2 \\
\hline Oral cavity & 2 \\
\hline Larynx & 1 \\
\hline Other & 1 \\
\hline \multicolumn{2}{|c|}{ No. of previous lines of systemic cancer therapy } \\
\hline 1 & 1 \\
\hline 2 & 5 \\
\hline$\geq 3$ & 10 \\
\hline \multicolumn{2}{|c|}{ Context of previous systemic therapy regimen no. } \\
\hline Primary disease & 4 \\
\hline Metastatic disease & 8 \\
\hline Primary + metastatic & 4 \\
\hline
\end{tabular}

Abbreviation: ECOG, Eastern Cooperative Oncology Group. 
The RR among nivolumab-treated patients was $12.0 \%$, including two partial responses by RECIST. The disease control rate was $81.2 \%$. Time to onset of pneumonitis ranged from 1 to 7 cycles (mean: 3.5 cycles), and time to onset of rash ranged from 2 to 6 courses (mean: 3.1 courses). No patients developed bacterial or viral pneumonia.

\section{PD-L1 Expression and p16 Status}

Tumor PD-L1 expression status and p16 status could be evaluated in 15 of 16 patients (93.7\%). Among these patients, 7 of 15 (43.7\%) had a PD-L1 expression level of $\geq 1 \%$.

Four of 15 patients were p16-positive.

\section{Safety}

The most common treatment-related AEs are shown in -Table 2.

The rate of treatment-related AEs was 68.7\%. There were no Grade 4 AEs. The most frequent AEs were fatigue, rash, and pruritus.

\section{Discussion}

The prognosis of patients with RM-SCCHN cancer after platinum-based chemotherapy is very poor. ${ }^{3}$

Nivolumab is the first standard treatment for RM-SCCHN within 6 months after platinum treatment.

Nivolumab demonstrated favorable OS, PFS, QOL, and RRs compared with standard chemotherapy for platinum-refractory disease in a global phase III trial. ${ }^{4}$

In March 2017, nivolumab was approved for platinumrefractory patients in Japan and has been used to treat recurrent metastatic squamous-cell carcinoma of the head and neck. Zargar et al in Canada questioned its cost-effectiveness, although they pointed out that a $20 \%$ reduction in price would lower the willingness-to-pay threshold. ${ }^{7}$ Tringale et al, looking at the CheckMate 141 trial, found it to be lacking in cost-effectiveness. ${ }^{8}$ In Japan, the potential indications for nivolumab are under discussion.

Nivolumab is a very expensive drug and we have to select the patients who could benefit the most, possibly based on

Table 2 Treatment-related adverse events in the patients (all were Grade 1-3)

\begin{tabular}{|l|l|l|}
\hline & $\begin{array}{l}\text { All grades, } \\
\boldsymbol{n}(\%)\end{array}$ & $\begin{array}{l}\text { Grade 3, } \\
\boldsymbol{n}(\%)\end{array}$ \\
\hline Any event & $11(68.7 \%)$ & $3(18 \%)$ \\
\hline Pneumonitis & $7(43 \%)$ & $1(6 \%)$ \\
\hline Rash & $6(37.5 \%)$ & $2(12 \%)$ \\
\hline Pruritus & $5(31 \%)$ & 0 \\
\hline $\begin{array}{l}\text { Kidney dysfunction } \\
\text { (elevated creatinine and/ } \\
\text { or hematuria) }\end{array}$ & $3(25 \%)$ & 0 \\
\hline Fatigue & $3(25 \%)$ & 0 \\
\hline Anorexia & $1(6 \%)$ & 0 \\
\hline $\begin{array}{l}\text { Endocrine disorder } \\
\text { (hypothyroidism) }\end{array}$ & $2(12 \%)$ & 0 \\
\hline
\end{tabular}

QOL years. Numerous searches for biomarkers suggestive of response to therapy are being performed ${ }^{9}$; there have been no other reports on the influence of PD-L1 status on prognosis.

In the Asian population, the incidence of skin reaction was higher than the global mean. ${ }^{6}$ Our patients tended to suffer an increased incidence of recurrent skin reactions, pneumonitis, and renal dysfunction compared with the global mean, although all were Grade 1 to 2 . The high pneumonitis incidence may have been due to the high proportion of heavy smokers in our study.

Given the special immune-related AEs associated with monoclonal antibodies, ${ }^{10,11}$ a multidisciplinary approach to treat them and to optimize therapeutic effectiveness is needed. Being able to select patients most likely to respond may make this treatment more cost-effective.

\section{Limitations}

The limitation of this study is its retrospective nature and the small number of subjects.

\section{Conclusion}

Our retrospective trial showed marginal improvement in survival in a small cohort of Japanese patients. It remains to be determined which subsets of patients will benefit from this treatment to the point that it is cost-effective.

Conflict of Interest

None declared.

\section{References}

1 Ferlay J, Soerjomataram I, Dikshit R, et al. Cancer incidence and mortality worldwide: sources, methods and major patterns in GLOBOCAN 2012. Int J Cancer 2015;136(05):E359-E386

2 Vermorken JB, Mesia R, Rivera F, et al. Platinum-based chemotherapy plus cetuximab in head and neck cancer. N Engl J Med 2008; 359(11):1116-1127

3 Saloura V, Cohen EE, Licitra L, et al. An open-label single-arm, phase II trial of zalutumumab, a human monoclonal anti-EGFR antibody, in patients with platinum-refractory squamous cell carcinoma of the head and neck. Cancer Chemother Pharmacol 2014;73(06):1227-1239

4 Ferris RL, Blumenschein G Jr, Fayette J, et al. Nivolumab for recurrent squamous-cell carcinoma of the head and neck. N Engl J Med 2016; 375(19):1856-1867

5 Harrington KJ, Ferris RL, Blumenschein G Jr, et al. Nivolumab versus standard, single-agent therapy of investigator's choice in recurrent or metastatic squamous cell carcinoma of the head and neck (CheckMate 141): health-related quality-of-life results from a randomised, phase 3 trial. Lancet Oncol 2017;18(08):1104-1115

6 Kiyota N, Hasegawa Y, Takahashi S, et al. A randomized, openlabel, Phase III clinical trial of nivolumab vs. therapy of investigator's choice in recurrent squamous cell carcinoma of the head and neck: a subanalysis of Asian patients versus the global population in checkmate 141. Oral Oncol 2017;73:138-146

7 Zargar M, McFarlane T, Chan KKW, Wong WWL. Cost-effectiveness of nivolumab in recurrent metastatic head and neck squamous cell carcinoma. Oncologist 2018;23(02):225-233 
e10 Nivolumab in Platinum-Refractory RM-SCCHN Yamazaki et al.

8 Tringale KR, Carroll KT, Zakeri K, Sacco AG, Barnachea L, Murphy JD. Cost-effectiveness analysis of nivolumab for treatment of platinumresistant recurrent or metastatic squamous cell carcinoma of the head and neck. J Natl Cancer Inst 2018;110(05):479-485

9 Maleki Vareki S, Garrigós C, Duran I. Biomarkers of response to PD-1/ PD-L1 inhibition. Crit Rev Oncol Hematol 2017;116:116-124
10 Lo Nigro C, Denaro N, Merlotti A, Merlano M. Head and neck cancer: improving outcomes with a multidisciplinary approach. Cancer Manag Res 2017;9:363-371

11 Naidoo J, Cappelli L, Lipson E, et al. A multidisciplinary toxicity team for cancer immunotherapy-related adverse events. J Clin Oncol 2018;36:6538-6538 